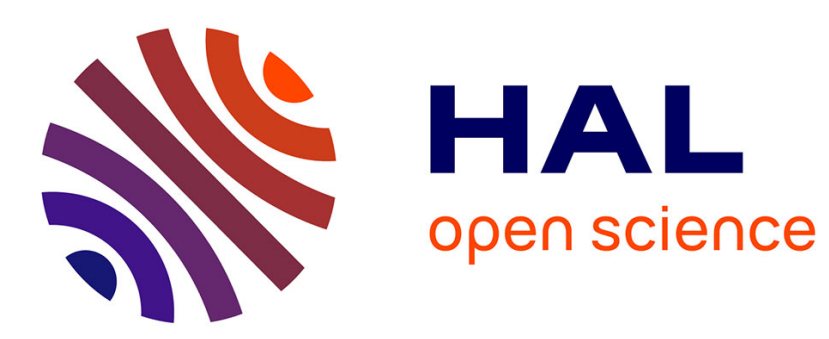

\title{
How is SCTP Able to Compete with TCP on a QoS-aware Satellite Network?
}

Lionel Bertaux, Thierry Gayraud, Pascal Berthou

\section{To cite this version:}

Lionel Bertaux, Thierry Gayraud, Pascal Berthou. How is SCTP Able to Compete with TCP on a QoS-aware Satellite Network?. Advances in Satellite and Space Communications (SPACOMM), Jun 2010, Athens, France. 10.1109/SPACOMM.2010.20 . hal-00533243

\section{HAL Id: hal-00533243 \\ https://hal.science/hal-00533243}

Submitted on 5 Nov 2010

HAL is a multi-disciplinary open access archive for the deposit and dissemination of scientific research documents, whether they are published or not. The documents may come from teaching and research institutions in France or abroad, or from public or private research centers.
L'archive ouverte pluridisciplinaire HAL, est destinée au dépôt et à la diffusion de documents scientifiques de niveau recherche, publiés ou non, émanant des établissements d'enseignement et de recherche français ou étrangers, des laboratoires publics ou privés. 


\title{
How is SCTP Able to Compete with TCP on a QoS-aware Satellite Network?
}

\author{
Lionel Bertaux $^{1,2}$, Thierry Gayraud ${ }^{1,2}$, Pascal Berthou ${ }^{1,2}$ \\ ${ }^{1}$ CNRS ; LAAS ; 7 avenue du Colonel Roche, F-31077 Toulouse, France \\ ${ }^{2}$ Université de Toulouse ; UPS, INSA, INP, ISAE ; LAAS ; F-31077 Toulouse, France \\ \{lbertaux, gayraud, berthou\}@laas.fr
}

\begin{abstract}
Transport protocols have been developed at first for terrestrial networks, but they are also used on communication satellite networks which have specific characteristics such as low bandwidth, QoS architecture and large latency. TCP and SCTP have been intensively studied in several circumstances, recommendations have been provided and results obtained. We are going to use the research done in order to study SCTP and TCP behavior on a satellite network implementing QoS architecture. Our intentions are to explain and predict the behavior of the transport protocols on such networks. The settings of these transport protocols will be studied in order to prove how they can be both improved on a satellite network with a $\mathrm{QoS}$ architecture.
\end{abstract}

Keywords - satellite communication networks; Quality of Service; transport protocols.

\section{INTRODUCTION}

In a previous paper we presented a simulation model of a DVB-S2/RCS Satellite network [1]. The assets of this model is a Quality of Service (QoS) architecture with an encapsulation mechanism taking into account the overhead introduced by the fragmentation from IP packets to MPE and ATM frames. In order to check its performances, two types of traffic were generated, one with time constraints: Real Time flows (RT), and the other one without constraints: non Real Time flows (n-RT). The main goal was to assure the best services to RT flows and to have a model as close as possible from a real QoS architecture. The analysis was done by studying the delays and the throughput of the several flows, and we concluded the model was efficient in simulating a communication satellite network with QoS architecture.

In addition to the tests done on this model, it seems interesting to compare the behavior of two transport protocol and to check if their behavior under QoS constraints fit with the studies already done on other networks. Stream Control Transmission Protocol (SCTP) is a quite recent transport protocol and it will be compare to the ubiquitous Transmission Control Protocol (TCP). Naturally developed for modern networks and current needs, SCTP got interesting features which explain the enthusiasm for this protocol. The simulation allows to obtain new measures and to anticipate on the implementation of SCTP over satellite networks by checking its response to an unfriendly environment.

Along with these experiments, we will demonstrate the influence of the transport protocol settings on the network performances: throughout, delay, buffer level, etc.
The first part of this paper summarizes the structure of DVB-S2/RCS satellite networks. The second focused on TCP and SCTP with their similarities and their behavior under specific circumstances. The third part introduces the model with its architecture and its parameters. The fourth part is dedicated to the simulations by explaining the choices made for the topology and the traffic generation before giving illustrations of the results obtained. The last part concludes on the foreseeable nature of the results, the ability of SCTP to compete with TCP and how relevant the improvements done are.

\section{SATELLITE NETWORKS}

\section{A. Communication satellite system}

Satellite networks have specific characteristics like a long delay and a small bandwidth. In order to guarantee better performances to some flows, a QoS architecture is implemented on such systems.

In a modern satellite network (Fig. 1), we can consider three main components: the Network Control Center (NCC) allocating the bandwidth on demand, the Gateway (GW) allowing an access to Internet, and several satellite terminals (ST) connected to an access network. The geostationary satellite links up together these components and by being connected to one of the access networks, users will be able to use Internet services and to communicate one with another.

At first, satellite communication networks were only able to establish unidirectional communication from the server to the client, supporting mainly TV broadcasting. However, as Internet is currently the reference network, using satellite networks as an access network seems interesting and the need of a return channel became obvious in order to allow a bidirectional communication. The protocol Digital Video Broadcast / Return Channel via Satellite (DVB/RCS) described in [3] was developed in order to complete the Digital Video Broadcasting via Satellite (DVB-S). On the forward link (from satellite to terminals), the bandwidth is really large (about 60Mpbs) and all the communications can be transmitted without problem, but on the return link (from terminals to satellite) the bandwidth is ten times smaller, so the Method Access Control (MAC) and the method used to allocate it over the terminals will drastically affect the connections. The several possibilities will be discussed in the next sub section. 


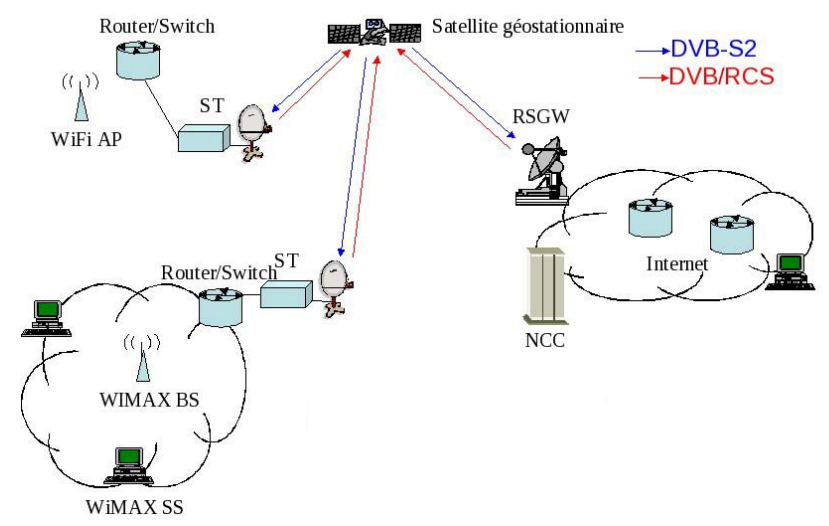

Figure 1. A DVB-S2/RCS satellite network

The increase of private connections bandwidth from $56 \mathrm{kbps}$ to more than $10 \mathrm{Mbps}$ in the last few years allowed the use of new applications such Voice on IP, Video on Demand, on-line games, etc. These services need a short delay and a short presentation delay unlike FTP connections that just need some bandwidth. Therefore a differentiation between the various flows is needed; they are classified in RT services and n-RT services. QoS architecture is used in satellite networks in order to implement that classification and allows integrating them with the technology used in the core networks of Internet: Differentiated Services (DiffServ).

\section{B. Encapsulation, Bandwidth Allocation and QoS Architecture}

The frames passing around the network are Asynchronous Transfer Mode (ATM) cells on the return channel and MPEG2-TS on the forward link. The access networks located beyond the ST and the Internet networks are using IP packets. In order to allow communication between these networks, encapsulation and fragmentation is necessary. This is done by means of ATM Adaptation Layer 5 (AAL5) and Multi Protocol Encapsulation (MPE). The sizes of ATM and MPEG2-TS frame are respectively 53 bytes and 188 bytes. Having a fixed size will make the calculation of the sending time for one frame easier, and this last parameter will only depend on the available bandwidth. But the overhead introduces by those two mechanisms is consequent (around 10\%) and can alter the comportment of TCP by changing the reactions of the congestion window. This mechanism is rather important and needs to be represented.

As explained earlier, the bandwidth of the return channel cannot be wasted. The geostationary satellite networks have a consequent delay (about $120 \mathrm{~ms}$ ) and if collision occurs this will result in a delay bigger than $300 \mathrm{~ms}$. In DVB-S/RCS satellite systems, a Time Division Multiple Access (TDMA) method is employed, the temporal slots are assigned to terminals and they can emit the frames without any risk of collision. To obtain a bigger bandwidth, Frequency Division Multiple Access (FDMA) is also used.
The easiest way to allocate these slots is to statically split them over all the terminals and according more slots to the one used as GW. This approach is efficient for a small number of terminals, but the bandwidth is not allocated according to the needs of the users. The Demand Assignment Multiple Access algorithm offers the possibility to allocate slots in two ways:

- Statically : Constant Rate Assignment (CRA)

- Dynamically : Rate or/and Volume Based Dynamic Capacity ( RDBC or/and VBDC)

Each terminal owns at least one CRA slot and send request to the NCC if it needs more. Depending on all the requests, the NCC responds to each terminal with the new allocation. The requests are sent periodically so the unused bandwidth can be split over the remaining ST needing it. They are compute by checking the level of the buffer located between Link and MAC layers. With the response, the MAC can send the frames at the determined time, so they fit in the slots.

By providing Internet access via the satellite network, traffic types are mixed even if they have various characteristics and requirements. In order to have a differentiation between services and to guarantee a Quality of Service, communication networks implement a specific architecture. With the DiffServ model, the routers won't act any more on the packets, but they blend them and compute the flows, allowing the network to easily guarantee QoS for some of them while the others are processed in a Best Effort way. With a similar architecture, flows can be differentiated at the IP layer in the ST with three different buffers Expedited Forwarding (EF), Assured Forwarding (AF) and Best Effort (BE). The frames are also classified between MAC and Link layer by using two distinct queues: Real Time (RT) and non-Real Time (n-RT). This architecture introduces a Quality of Service and allows a differentiation between the packets.

\section{TCP AND SCTP}

Since the beginning of Internet, TCP is intensively used for several reasons such as: reliability, congestion avoidance algorithm, flow control, packet delivery order scheduling and others. Enhancements have been done to improve its efficiency via new implementations of which main releases are: Tahoe, NewReno, Vegas, etc. These features allowed TCP to overcome the matters introduced by the modern uses of Internet and the heterogeneity of current networks. But TCP doesn't use the full potential of present and future communication systems, therefore some researchers are working on a new protocol in order to supplant it.

For nearly ten years now, SCTP has been designed, studied and can already be implemented on personal computers. Besides having most of the components of TCP, SCTP has new features fulfilling the needs of modern Internet traffic and new ways to get to it: multi streaming and multi homing. In SCTP, a communication between two end users is no more called a connection but an association.

The multi streaming option allows having more than one stream in an association; it can be used to handle the sending 
of several files at the same time. If some parts of a file are lost, the chunks of the others files are still sent on the network and the lost segments are retransmitted later.

The multi homing capability improves the reliability of associations by taking into account the many interfaces that can be used to get access to a network. This allows for example the use of both wired and wireless connections at the same time. One interface is used as a primary path and the second path is used for retransmission in case of error or failure. After a given number of errors or Retransmission Time Out (RTO), the second path can be selected as primary. It can also be settled so both interfaces are used, providing more bandwidth (Concurrent Multipath Transfer).

In both protocols, the outgoing traffic is managed by a congestion window (CWND). It sets a number of bytes (for SCTP) or packets (for TCP) that can be sent and not yet acknowledged, but instead of TCP that process the CWND at sender side, SCTP calculates it on the receiver side and pass the information to the sender through the acknowledgments (ACK). In order to estimate the network load, two different algorithms are used to increase the congestion window: slow start at the beginning of the communication and congestion avoidance after a given threshold. In the slow start phase, the CWND is increased approximately by one Maximum Transmission Unit (MTU) for every positive ACK while in congestion avoidance, it is increased by one MTU every Round Trip Time (RTT). When a retransmission is needed, the CWND is decreased to one MTU in case of timeout or to the threshold in case of fast retransmission (if enabled).

Besides Multi-Homing and Multi-Streaming, SCTP has another advantage on TCP, that is the potential use to replace User Datagram Protocol (UDP) so just one protocol is needed for the transport layer, and unlike UDP which is not connection-oriented, SCTP will establish the association first. The reliability of the connections is increased by checking if the receiver is ready or not. The inconvenience being waiting one RTT before the connection can be established.

The simulation of TCP and SCTP under QoS constraints will drastically improve their accommodation capabilities on a network with low bandwidth and long delay. Studies on the behavior of SCTP and TCP on satellites networks have been done [4], [5], proving they can be adapted to this kind of situation and recommendations have been made on the settings of TCP [6], [7] on Mobile Ad Hoc-NETworks.

Taking that into account, the analysis will focus on the comparison between measures with and without settings, how theses results can be anticipated and used to improve the reliability and the performance of the network. The simulation chapter takes a deeper look at the setting that can be applied on the two transport protocols, while the next section introduces the model and its characteristics.

\section{MODEL PRESENTATION}

To simulate the satellite network, the software Network Simulator 2 was chosen [7]. Its modularity as well as its capacity to simulate Next Generation Networks (NGN) allowed him to being used by researchers before making an implementation on a real system.

The model is based on the one presented in [8], and is improved by adding a QoS architecture and taking into account the overhead introduced by encapsulations (IP over ATM and IP over MPEG-2TS). In order to implement these features, modifications were done at two levels:

- $\quad$ RT and n-RT files between MAC and Link layers,

- $\quad \mathrm{EF}, \mathrm{AF}$ and BE files at the Network layer.

Both changes work on the same way. Several queues are used to store flows with different priority levels, where packets are stored and picked up depending on their FID (Flow IDentification field of transport protocol header). A manager is located beyond theses queues and is in charge of choosing the right file.

Between MAC and Link layer, a simple queue contains packets before they are sent to the physical support. Sorting packets at this level is important and will have a significant impact on the QoS. This single queue has been removed and replaced by two queues called RT and n-RT. The RT queue have a higher priority than the n-RT one, thus it contains only the flows with high QoS constraints. The manager waits for MAC to be idle and checks the queues level. The packets waiting in n-RT file are picked only when the RT file is empty and just one can be transmit before calling again the manager.

Applied on most of the edge and core routers of the Internet network, the DiffServ architecture allows merging packets in flows and simplifying the process done by the core nodes. The packets are stored in three different queues depending on their flow ID. This classification is done at IP layer, so by choosing the file from which packets are unqueued, the system makes a scheduling above the two MAC queues introduced previously.

Unlike the wired links in NS-2, the satellite links do not contain buffers, and we cannot use the DiffServ function available in the basic version of the simulator. To overcome this issue, this feature has been implemented between the Encapsulation agent and the entry of the node.

Several types of traffic will be stored in these queues; they are sorted in three types of services:

- Expedited Forwarding (EF) needing low delay,

- Assured Forwarding (AF) needing a minimum bandwidth,

- $\quad$ Best Effort (BE): other flows.

The first type of service fits with the RT requirements, and the two others will go through the n-RT queue at the MAC layer. In our model, the packets arriving in a node are sorted and stored in the queues, where they stay while the MAC buffer level is above a given level. This way, we will able to prevent the RT and n-RT queues to overflow and to avoid unnecessary loss. The check is performed by a manager called every time a packet is queued or when a packet is dequeued from the MAC queues. 
The frames on the satellite network are MPEG2-TS on the forward link and ATM on the return link while in the access networks IP packets are traveling. On the real system, an encapsulation and a fragmentation are made in the STs, but modeling the real fragmentation is not possible due to the limits of the simulator. To overcome this issue, when a packet arrived in a terminal, its size is changed by adding the overhead corresponding to the successive encapsulations from IP to ATM. In the satellite, the packet size is changed to fit to the encapsulation from IP to MPEG2-TS, and when it goes out from the arrival terminal, the original size of the IP packet is put back on. These mechanisms are done by new agents located in the Satellite Terminals. In order to rout the packets from the entry of the node to the right agent, a new specific classifier is inserted between entry point and the address classifier.

\section{Simulations}

The simulation chapter is organized in two parts. The first sub-section is a presentation of the model and the network, while the second part focuses on the transport protocols.

\section{A. Presentation}

As a real satellite system, the model has a QoS architecture that allows flow differentiation and scheduling, an encapsulation mechanism that simulates the overhead introduced by the transition from packets to frames and a DAMA algorithm allowing a dynamic allocation of the bandwidth. The topology (Fig. 2) has also been chosen to symbolize a real satellite network:

- The Geostationary Satellite,

- The Gate Way (GW) located beyond the Network Control Center (NCC),

- The Satellite Terminals (ST).

The end users are connected to the ST with a wired link, but having a wireless connection is also possible. The uplink bandwidth allocate to each terminal is set according to the Satsix platform used at LAAS: $128 \mathrm{kbps}$ in CRA and $128 \mathrm{kbps}$ in RBDC.

In order to observe the efficiency of the QoS architecture, two types of traffic with different priority level have been generated: CBR and FTP, respectively Expedited Forwarding and Assured Forwarding. It was done so to guarantee a low delay with a few losses to the CBR traffic and to allow the FTP connection to being established and to have a throughput as large as possible. The contest between the flows will force the CWND to adapt its rate depending on the remaining bandwidth available.

The comparison between SCTP and TCP/UDP behaviors allows us to obtain new measures on the delay and the throughput and to check how consistent the results are.

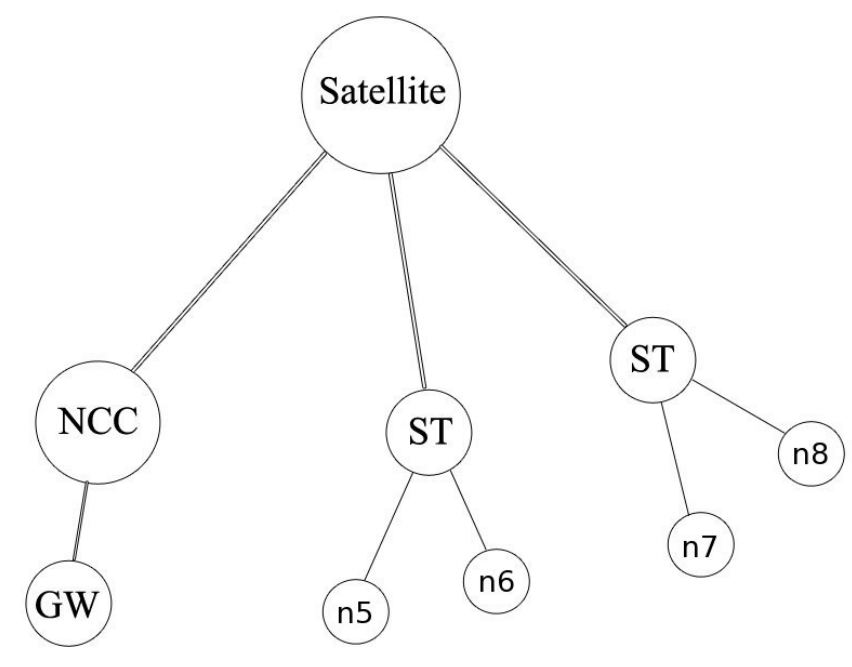

Figure 2. Topology of the network simulated for this paper

\section{B. Settings of Transport Protocols}

The impact of parameters setting has been introduced in section 3. The following parameters can be settled on the transport layer protocols:

- Return Time Out (RTO): initial, minimal, maximal.

- Congestion window (CWND): Maximum size, threshold maximum value.

This study focused on the congestion window and threshold, two particular cases are:

- Parameters with default values,

- Evaluation of parameters settled depending on the environment.

The parameters settled with high values are big enough so the threshold and the maximum congestion window cannot be reached and we will be able to observe the accommodation of the transport protocols to the network restrictions. In this case, the simulations were run by starting all connections at the same time.

In order to adapt values to the environment, several simulations were done with the maximal threshold and the maximal congestion window changing from 5 to 80 packets. The simulations last 1000 seconds, FTP starting at time 0 and CBR at 400 seconds. To avoid slow start phase at the beginning and at the introduction of $\mathrm{CBR}$, the measures focus on two intervals: from 50 to 350 seconds for single FTP, and from 600 to 900 seconds for FTP + CBR. During theses intervals, traffic is in a stable state so the mean value is quite representative. The overall results will be also discussed to conclude on the efficiency of the settings.

\section{Simulations overview}

This section contains the results of the simulations done the model. All the results will be analyzed in the next part. 
The first simulation was done with SCTP and TCP with default value. Types of traffic generated are CBR and FTP with different priority levels. Below pictures show the behavior of TCP and SCTP without limitations. Fig. 3 shows the congestion window size in packets, Fig. 4 shows the delay suffered by the connections and the RTO value of the transport protocols. Buffers' level is not noticed here since they fluctuate according to the congestion window size. The throughput of the FTP connections is always equal to the bandwidth offered by the network. The illustration ends at 200 seconds since the traffics behavior will just repeat over and over (CBR connections ended at 110 seconds).

In a second time, simulations with limitations on maximum CWND size and maximum threshold were made. The results on the threshold limitation won't appear in this paper since they are not really relevant: once the maximal threshold is reached, the congestion window can still increase and the sending rate will still be over-sized compared to the available bandwidth.

Fig. 5 illustrates the simulations' results when the maximum values of congestion window are changed. It shows the average delay of FTP connections without (curves "sctp" and "tcp") and with a CBR connection set at higher priority (curves "sctp cbr" and "tcp cbr"). The average throughput of FTP connections and average buffers level of are presented in Table 1. The measures in this table were done without CBR connections: the buffers' levels are quite similar in the two cases.

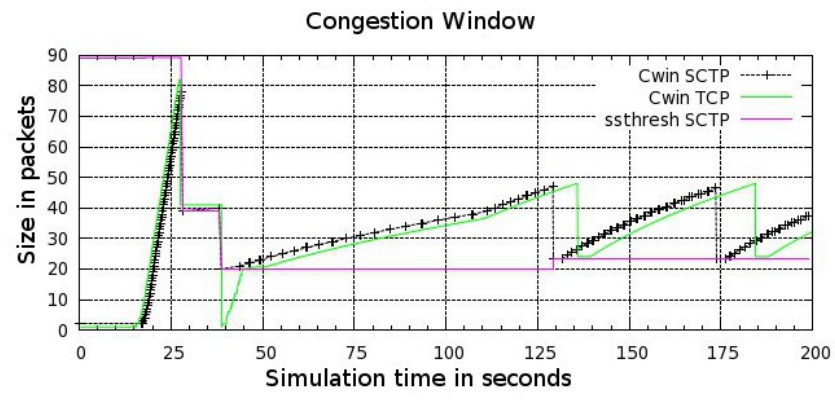

Figure 3. Congestion window size of SCTP and TCP with default values of congestion window

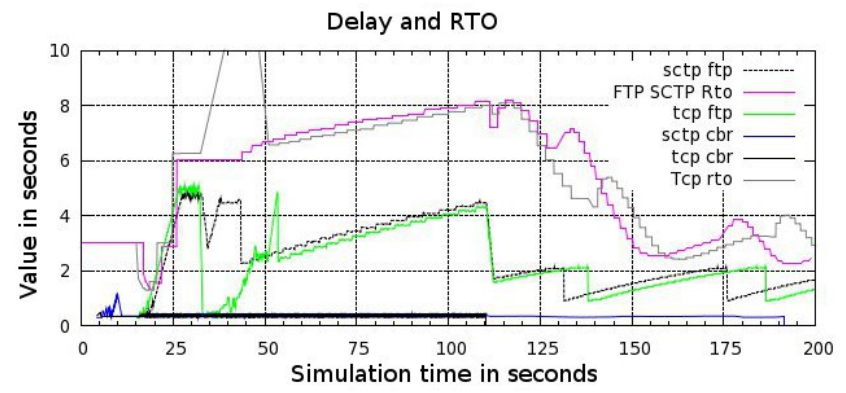

Figure 4. Average delay of FTP and CBR connections with default values of congestion window

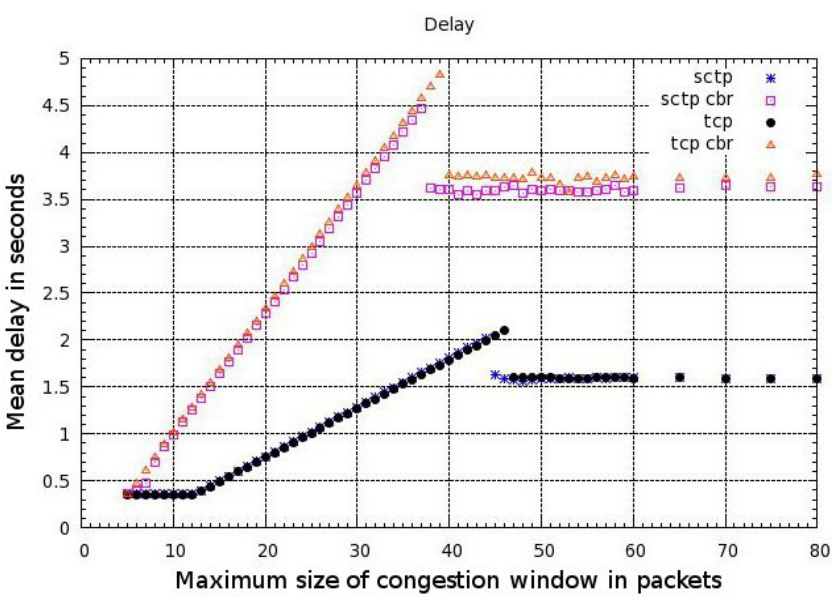

Figure 5. Average delay of the FTP connections depending on the maximum congestion window size

TABLE I. AVERAGE THROUGHPUT AND BUFFERS' LEVEL DEPENDING ON MAXIMUM CONGESTION WINDOW SIZE

\begin{tabular}{|c|c|c|}
\hline \multirow{2}{*}{$\underset{\text { CWND }}{\operatorname{Max}}$} & \multicolumn{2}{|c|}{ Average value } \\
\hline & $\begin{array}{c}\text { Throughput in kilo Bytes per } \\
\text { seconds }(T C P / S C T P)\end{array}$ & $\begin{array}{l}\text { Buffers' level in kilo } \\
\text { Bytes (TCP / SCTP) }\end{array}$ \\
\hline 5 & $11.5 / 11.4$ & $0.8 / 0.8$ \\
\hline 10 & $23.0 / 22.8$ & $0.8 / 0.8$ \\
\hline 20 & $28.5 / 28.3$ & $13.5 / 13.4$ \\
\hline 30 & $28.5 / 28.3$ & $30.2 / 29.8$ \\
\hline 40 & $28.5 / 28.3$ & $46.8 / 46.2$ \\
\hline 50 & $28.4 / 27.8$ & $39.1 / 40.2$ \\
\hline 70 & $28.4 / 27.8$ & $39.1 / 40.2$ \\
\hline
\end{tabular}

A chaotic behavior of both protocols can be observed with a limitation of the congestion window around 40 packets: the delay suffered by the communication is the largest, even compared to higher limitation. This phenomenon and the other results will be analyzed in the last part of this paper.

\section{ANALYZE}

The previous section shows the results obtained from simulations with different settings applied to the transport protocols on a DAMA QoS model of a communication satellite network. Our aim being comparing TCP and SCTP efficiency and analyzing the influence of parameters settings, the measures description and analysis is going to focused on the transport protocols behavior.

As expected, the behavior of the two transport protocols are similar (see Fig. 3 and Fig. 4), since they use the same mechanisms to manage their sending rate (CWND with slow start and congestion avoidance). It is clearly visible that delays (Fig. 3) suffered by FTP connections fluctuate along with the congestion window size (Fig. 4). This phenomenon 
is due to buffers getting filled up and to the throughput reaching the limited amount of bandwidth available (less than $32 \mathrm{kB}$ and less than $16 \mathrm{kB}$ when there is $\mathrm{CBR}$ ). When buffers overflow (at 25 seconds for instance), packets are dropped and after one RTO congestion windows fall down at threshold and starts to send again. But buffers are not empty yet and last send packet is not sure to arrive before RTO. If a timeout occurs again, the congestion windows will drop at its minimum value, and the same scheme will start again.

Before going deeper in the analysis, it is relevant to notice that all measures are done between sender and receiver transport layers. Buffers located between application and transport layer are not taking into account, therefore the delays reported in the previous section are not exactly the ones suffered by applications, but they affect them earnestly.

Since the bandwidth available is restricted, the transport protocol doesn't need to be able to sent data at high rate. Therefore, regulate the size of maximal congestion window to the size of the network could be a solution to buffer overflow resulting in loss and high latency.

On Fig. 5, the average delay depending on the maximum CWND size is fluctuating from less than 0.5 seconds up to 1.5 seconds without CBR and up to 3.5 seconds with CBR. Table 1 resumes the measurements made on throughput and buffers levels. It displays mean values depending on the maximum CWND size. From a 20 packets size, the optimum throughput is reached and buffers are not full while delay is below 1 second. While there is higher priorities flows buffers' levels are nearly the same, but the available bandwidth is drastically reduced and so is the throughput. Nevertheless, it has the same values as the first case, without restrictions.

As noticed in the previous section, the limitation of the CWND maximal size around 40 packets results in a remarkable behavior. The delays and the buffers levels are the highest for both protocols. This is due to a simple fact: at this rate, the network capacity reaches its limits. The amount of data sent can be handled by the buffers, so there is no overflow and the mean congestion window size is equal to the limitation. If the limitation is high enough, loss occurs in the buffers and the CWND is decreased. This result is interesting and leads to a first conclusion: the modifications done have a resulting impression on the transport protocols behaviors and have to be done carefully in order not to degrade the network efficiency.

From these results, we can conclude that SCTP and TCP have a similar behavior under QoS constraints and both are disturbed at the same values: stable congestion window size leading to remarkable results. SCTP is a quite efficient transport protocol compared to TCP, but its performances are nearly the same without the use of its new features such as multi-homing and multi-streaming. By adding these components, no comparison can be made between the two protocols, since TCP won't be able to switch from one network to another so easily.

It is significant that regulating the maximum sending rate of a transport protocol improves average delay and decreases the buffers' level, without decreasing the throughput.

\section{CONCLUSION}

Satellite networks seem to be fit for Internet access in wide areas, but the constraints they introduce are hard to overcome. The transport protocols used in these systems were first designed for wired networks or wireless networks with a low delay, they quickly show their limitations and have to be implemented carefully.

The analysis demonstrates the similarities between the protocols and how SCTP is able to compete with TCP on satellite networks. Furthermore, the new features of SCTP could be used in such networks to improve the reliability and the efficiency of the communication. Our proposition has already been thought for wired networks but what we intend to do is showing how significant such care can improve communications over satellite networks.

The solution simulated is one of many, but merged with others implementations (both on hardware and software), it can adapt the satellite networks to future needs and communications. The future efficiency of satellite networks will not only depend on the architecture or on the bandwidth available: transport protocols have to be aware of the network below or to be settled for such systems. The enduser also needs a properly configured station in order to take advantage of the architecture and on the protocols developed.

Since this study was more qualitative than quantitative, the results are just a clue on what can be done in protocols configuration to improve performances. The next step would be having a quantitative approach of the limitation on the congestion window, so a link can be established between the bandwidth available, the size of the buffers, the maximal sending rate and the limitations. These results could lead to an implementation on the test bed Platine from LAAS-CNRS in order to complete the simulations before being tested on real Satellite Terminals.

\section{REFERENCES}

[1] T. Gayraud, L. Bertaux, and P. Berthou, "A NS-2 simulation model of DVB-S2/RCS satellite netwok", $15^{\text {th }} \mathrm{Ka}$ Band Conference, (KACONF 09), Italy.

[2] H.Skinnemoen, R.Leirvik, J.Hetland, H.Fanebust, and V.Paxal. "Interactive IP-network via satellite DVB-RCS". DVB-RCS Dept., Nera Broadband Satellite \& Nera SatCom AS, Billingstad, Norway : April 2004

[3] G. Neglia, V. Mancuso, F. Saitta, and I. Tinnirello, "A simulation study of TCP performances over satellite channels", $34^{\text {th }}$ COSPAR Scientific Assembly, Houston, 2002.

[4] S. Fu and M. Atiquzzaman, "SCTP over satellite networks", in IEEE Computer Communications Workshop (CCW 03).

[5] K. Chen, Y. Xue, and K. Nahrstedt, "On setting TCP's congestion window limit in mobile ad hoc networks", in IEEE International Conference on Communications 2003 (ICC 2003).

[6] K.W. Kim, P. Lorenz and M.M.O Lee, "A new tuning maximum congestion window for improving TCP performance on MANET", in Systems Communications, 2005. Proceedings Volume, pages 73-78.

[7] VINT project. "The Network Simulator - ns-2". http://nsnam.isi.edu/nsnam/index.php/Main_Page.

[8] A. Gotta, F. Potorti, and R. Secchi. "Simulating Dynamic Bandwidth Allocation on Satellite Links." University of Genoa, 2006. 